\title{
Vascular quality of care pilot study: how admission to a vascular surgery service affects evidence-based pharmacologic risk factor modification in patients with lower extremity peripheral arterial disease
}

This article was published in the following Dove Press journal:

Vascular Health and Risk Management

4 June 2014

Number of times this article has been viewed

\author{
Naomi Steenhof $f^{1,2}$ \\ Francesca Le Piane' \\ Kori Leblanc ${ }^{1-3}$ \\ Naomi R Eisenberg ${ }^{4}$ \\ Yvonne Kwan' \\ Christine Malmberg ${ }^{1,6}$ \\ Alexandra Papadopoulos 5,7 \\ Graham Roche-Nagle ${ }^{4,7,8}$ \\ 'Department of Pharmacy, University \\ Health Network, ${ }^{2}$ Leslie Dan Faculty \\ of Pharmacy, University of Toronto, \\ ${ }^{3}$ Centre for Innovation in Complex \\ Care, University Health Network, \\ ${ }^{4}$ Division of Vascular Surgery, \\ University Health Network, ${ }^{5}$ Faculty \\ of Nursing, University of Toronto, \\ Toronto, ON, ${ }^{6}$ Victoria General \\ Hospital, Vancouver Island Health \\ Authority, Victoria, BC, ${ }^{7}$ Peter Munk \\ Cardiac Centre, University Health \\ Network, ${ }^{8}$ Faculty of Medicine, \\ University of Toronto, Toronto, ON, \\ Canada
}

Background: Peripheral arterial disease (PAD) guidelines recommend aggressive risk factor modification to improve cardiovascular outcomes. Recommended pharmacologic therapies include antiplatelets, angiotensin converting enzyme (ACE) inhibitors, and HMG-CoA-reductase inhibitors (statins).

Purpose: We studied the degree to which patient admission to a vascular surgery service increased the use of these therapies.

Patients and methods: The authors conducted a retrospective chart review of 150 patients with PAD admitted to the vascular surgery service at a large Canadian tertiary care hospital. The use of recommended pharmacologic therapies at the time of admission and discharge were compared. A multidisciplinary clinical team established criteria by which patients were deemed ineligible to receive any of the recommended therapies. Angiotensin receptor blockers (ARBs) were considered an alternative to ACE inhibitors.

Results: Prior to hospital admission, $64 \%$ of patients were on antiplatelet therapy, $67 \%$ were on an ACE inhibitor or ARB, and $71 \%$ were on a statin. At the time of discharge, $91 \%$ of patients were on an antiplatelet (or not, with an acceptable reason), 77\% were on an ACE inhibitor or an ARB (or not, with an acceptable reason), and $85 \%$ were on a statin (or not, with an acceptable reason). While new prescriptions were largely responsible for improved guideline adherence with antiplatelets and statins, most of the apparent improvement in ACE inhibitor and ARB use was the result of identifying an acceptable reason for not having them prescribed.

Conclusion: This hypothesis generating pilot study supports the findings of others that there is suboptimal prescription of pharmacologic risk reduction therapies in the PAD population. Admission to a vascular service increases these rates. Nevertheless, some patients are still not receiving evidence-based treatment at discharge even after consideration of acceptable reasons. Strategies are needed to improve PAD guideline adherence in both the community at large and the vascular surgery service.

Keywords: guideline adherence, vascular protection, risk reduction

\section{Introduction}

Peripheral arterial disease (PAD) is a prevalent condition that affects $12 \%$ to $29 \%$ of the elderly Canadian population ${ }^{1}$ and is associated with a high risk of cardiovascular morbidity and mortality. Patients with PAD have widespread arterial disease; consequently, the risk of stroke, myocardial infarction, and cardiovascular death
Correspondence: Naomi Steenhof Pharmacy Department, Toronto Western Hospital, UHN, 399 Bathurst St Toronto, ON M5T 2S8, Canada Tel + I $4166035800 \times 578$ I

Email naomi.steenhof@uhn.ca 
is significantly increased. ${ }^{2}$ PAD is also associated with decreased functional capacity, reduced quality of life, and limb amputation.

The 2005 American College of Cardiology/American Heart Association Guidelines for the Management of Patients with Peripheral Arterial Disease ${ }^{3}$ (updated in 2011), ${ }^{4}$ Canadian Cardiovascular Society 2005 PAD Consensus Document, ${ }^{5}$ and 2011 Canadian Cardiovascular Society Antiplatelet Guidelines ${ }^{6}$ recommend aggressive risk factor modification to improve patient outcomes. In addition to exercise, smoking cessation, and hypertension and diabetes management, the recommended risk reduction therapies for all patients (unless contraindicated) include an antiplatelet agent, an angiotensin converting enzyme (ACE) inhibitor, and an HMG-CoA-reductase inhibitor (statin).

Studies show that patients with PAD are suboptimally managed from a pharmacotherapy perspective, which is associated with increased all-cause mortality. ${ }^{7}$ Worldwide prescriber adherence to PAD guideline-based therapy is generally low; however, to our knowledge, only two studies have reviewed guideline adherence in Canada. ${ }^{8-9}$ An observational, retrospective study from Quebec evaluated a population-based cohort of patients with PAD who were discharged from a tertiary care teaching hospital and found that secondary prevention treatment remained suboptimal. ${ }^{8}$ Similarly, a recent retrospective pilot study out of Alberta determined that only about half of patients with diabetes who underwent vascular surgery received risk factor modification with all three recommended therapies. ${ }^{9}$

The influence of patient gender on the medical treatment of PAD has been investigated in several studies with differing results. One study from Ireland found similar prescription rates for ACE inhibitors in men and women. ${ }^{10}$ In contrast, the study from Quebec found that significantly more men than women used statins (49.1\% versus [vs] 45.6\%) and ACE inhibitors (44.5\% vs $39.3 \%$ ). Data on differences in treatment based on type of admission (elective vs emergent) is limited.

This retrospective chart review pilot study was designed to determine whether in-patient admission to a multidisciplinary vascular surgery service at a tertiary teaching hospital improved the number of patients who received pharmacological risk factor modification with antiplatelets, ACE inhibitors, and statins in patients with lower extremity PAD. We also examined the documented or presumed reasons for not prescribing these medications. Finally, we investigated whether there were differences in prescribing rates according to type of admission (elective vs emergent) and patient gender.

\section{Methods}

\section{Study setting}

Our hospital is a 1,000 bed tertiary care academic teaching center located in Toronto, Canada. Each year, 5,000 patients are referred for vascular surgery consultations and more than 800 major reconstructions are performed. ${ }^{11}$ The in-patient vascular service consists of approximately 17 beds and serves as a quaternary referral center for CritiCall Ontario, a 24-hour emergency referral service for physicians across Ontario. ${ }^{12}$ Patient care is provided by an interdisciplinary team, which includes surgeons, fellows, resident physicians, nurses, technologists, pharmacists, physical and occupational therapists, social workers, and dietitians. Patients access the service as emergency, urgent, or elective hospital admissions.

\section{Study population}

We screened all consecutive patients admitted to the in-patient vascular surgery service beginning in January 2010 until 150 patients met inclusion criteria. To be included, patients required a primary or secondary diagnosis of lower extremity PAD documented in the preadmission clinic notes or discharge summary. If the diagnosis was not specified, the patient was considered to have lower extremity PAD if admitted for the following interventions: aortofemoral bypass, axillaryfemoral bypass, femoral-femoral crossover, femoral-popliteal bypass, femoral-tibial bypass, amputation, and peripheral angiogram with or without angioplasty or stent.

Exclusion criteria included lack of diagnosis of lowerextremity PAD, death during hospital admission, lack of a comprehensive or best possible medication history (BPMH), or lack of a discharge summary that included medications at discharge. Patients were considered to have a BPMH if the electronic patient record contained a pharmacist-documented list of preadmission medications which involves interviewing the patient, checking multiple sources of information, and taking note of any issues with compliance. If a patient was admitted to the service more than once during the study period, only data from the first admission were analyzed.

\section{Study design}

The institution's Research Ethics Board approved the study protocol. The sample size of 150 patients was chosen based on the timeframe and resources available for this project. A standardized data collection form was created and used to retrieve data from the electronic patient record, including medications documented in the BPMH, medications listed on the discharge summary, patient demographics, and pertinent laboratory values. Documented comorbidities were collected from the discharge summary and preadmission 
clinic notes (if available). Rates of use of the following guideline-recommended classes of medications were extracted - antiplatelet agents, ACE inhibitors, and statins. Because angiotensin receptor blockers (ARBs) have similar benefits ${ }^{13}$ and in practice are often used as an alternative to ACE inhibitors, rates of use of ARBs were also extracted.

A list of clinically acceptable reasons for not prescribing specific agents was created and agreed upon by an expert multidisciplinary clinical team prior to the chart review. This team was comprised of a vascular surgeon, five pharmacists, and a nurse practitioner. Where consensus was not achieved, input from external experts was obtained. During data collection, if one clinically acceptable reason from this list was found, additional reasons were not sought.

Each patient admission was categorized as elective or emergent. The patients were classified as elective if they had been seen in the preadmission clinic or if the discharge summary indicated the reason for admission was an elective procedure. Patients were classified as emergent if they were admitted through the emergency department or transferred urgently from another facility for emergency vascular consultation.

\section{Endpoints}

The primary endpoint was the percentage of patients receiving guideline-based medications before and after admission to a vascular surgery service. Secondary endpoints included:
1) the list of clinically acceptable reasons for not prescribing specific medications, 2) the percentage of patients on all three of the recommended risk-modifying medication classes, and 3 ) the percentage of patients on risk-modifying medications stratified by type of admission (elective vs emergent) and patient gender.

Descriptive statistical analyses of all demographic variables and study endpoints were performed with the use of Excel ${ }^{\circledR}$ software, version 11 (Microsoft Corporation ${ }^{\circledR}$, Redmond, WA, USA).

\section{Results}

All patients admitted to the vascular surgery service from January 2010 to July 2010 were screened for eligibility. Figure 1 shows the selection of study population. Four hundred eighteen patients were screened sequentially until 150 patients met inclusion criteria. Table 1 shows the mean age was 71.4 years and $33.3 \%$ of the patients were women.

Results are documented in Table 2. Prior to hospital admission, $64 \%$ of patients were on antiplatelet therapy, $66.7 \%$ were taking either an $\mathrm{ACE}$ inhibitor or $\mathrm{ARB}$, and $71.3 \%$ were taking a statin. At the time of discharge, $82.7 \%$ of patients were discharged on an antiplatelet, $59.3 \%$ were discharged on either an $\mathrm{ACE}$ inhibitor or $\mathrm{ARB}$, and $82 \%$ were discharged on a statin. When including patients who were not

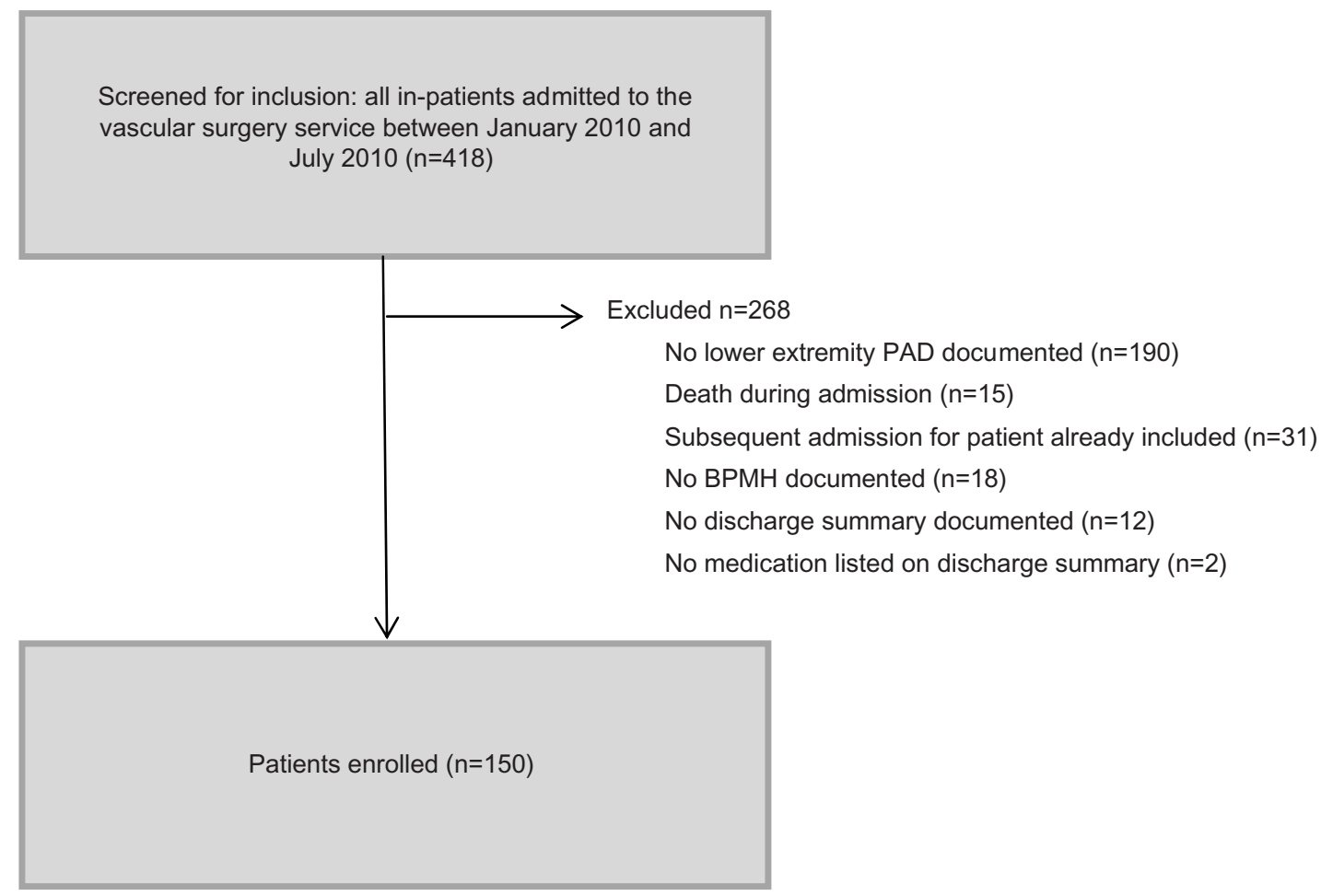

Figure I Selection of patients with lower-extremity peripheral arterial disease.

Abbreviations: BPMH, best possible medication history; PAD, peripheral arterial disease. 
Table I Baseline characteristics of PAD patients $(n=I 50)$

\begin{tabular}{|c|c|}
\hline Characteristic & n (\%) \\
\hline Age in years, mean (range) & $71.4(38-96)$ \\
\hline \multicolumn{2}{|l|}{ Age group } \\
\hline $38-64$ years & $39(26)$ \\
\hline $65-79$ years & $74(49.3)$ \\
\hline$>80$ years & $37(24.7)$ \\
\hline Female & $50(33.3)$ \\
\hline Length of stay in days, median (range) & $8(2-45)$ \\
\hline \multicolumn{2}{|l|}{ Type of admission } \\
\hline Elective & $70(46.7)$ \\
\hline Emergent & $80(53.3)$ \\
\hline Elective cases with documented PAC assessment & $54(77.1)$ \\
\hline Family doctor documented in discharge summary & $122(81.3)$ \\
\hline \multicolumn{2}{|l|}{ BPMH documented } \\
\hline On admission & I0I (67.3) \\
\hline Prior to admission & $49(32.7)$ \\
\hline \multicolumn{2}{|l|}{ Documented comorbidities } \\
\hline Cerebrovascular disease & $31(20.7)$ \\
\hline Coronary artery disease & $85(56.7)$ \\
\hline Diabetes & $61(40.7)$ \\
\hline Hypertension & $120(80)$ \\
\hline Hyperlipidemia & $59(39.3)$ \\
\hline Smoking history & $61(40.7)$ \\
\hline Chronic kidney disease & $33(22)$ \\
\hline
\end{tabular}

Abbreviations: BPMH, best possible medication history; PAC, preadmission clinic; $P A D$, peripheral arterial disease.

on therapy due to a clinically acceptable reason: $91.3 \%$ of patients were on an antiplatelet agent (or not, with an acceptable reason), $77.3 \%$ of patients were on an ACE inhibitor or ARB (or not, with an acceptable reason), and $85.3 \%$ of patients were on a statin (or not, with an acceptable reason) at discharge. Antiplatelet agents included acetylsalicylic acid (most commonly) and clopidogrel $75 \mathrm{mg}$ once daily.
The percentage of patients on all three classes of medications concurrently was $40.7 \%$ prior to admission. At discharge, $60.7 \%$ were on all three classes or had an acceptable reason not to be on therapy. Patients admitted electively had higher rates of guideline-based therapy both prior to admission and at discharge compared to patients admitted emergently.

The clinically acceptable reasons for not prescribing a guideline-recommended agent are listed in Table 3. The most common reason for not being on an antiplatelet agent (seven of 13 patients) was because the patient was on oral anticoagulation therapy for another indication (ie, atrial fibrillation) and had no other documented indication for antiplatelet therapy. ${ }^{6}$ The most common reason for not prescribing an ACE inhibitor or ARB was acute renal dysfunction (17 of 27 patients). The most common reasons for not prescribing a statin were allergy (two of five patients) and hepatic impairment (two of five patients).

An increase in prescription of all three classes of medications was observed in both males and females (Table 4). When comparing males and females, there were very similar rates of prescription of antiplatelets or ACE inhibitors/ARBs at discharge. Of particular interest was a trend to have a higher proportion of males (90\%) on a statin at discharge (or not, with a clinically acceptable reason) compared to females $(76 \%)$.

\section{Discussion}

We found that after in-patient admission to a vascular surgery service, more patients were discharged on certain guideline-

Table 2 Prescribed risk factor modification therapy, stratified by type of admission

\begin{tabular}{|c|c|c|c|c|c|}
\hline & $\begin{array}{l}\text { Admission } \\
\text { type }\end{array}$ & $\begin{array}{l}\text { Patient on class of } \\
\text { medication prior to } \\
\text { admission \% (n) }\end{array}$ & $\begin{array}{l}\text { Patient on class } \\
\text { of medication at } \\
\text { discharge \% (n) }\end{array}$ & $\begin{array}{l}\text { Patient on class of } \\
\text { medication at discharge, } \\
\text { or not on medication } \\
\text { with reason } \%(n)\end{array}$ & $\begin{array}{l}\text { Percent } \\
\text { improved }^{b}\end{array}$ \\
\hline Antiplatelet & All $(n=150)$ & $64(96)$ & 82.7 (I24) & 91.3 (I37) & +27.3 \\
\hline Elective $(n=70)$ & & 77.1 (54) & $81.4(57)$ & $91.4(64)$ & +14.3 \\
\hline Emergent $(n=80)$ & & $52.5(42)$ & $83.8(67)$ & $91.3(73)$ & +38.8 \\
\hline ACEI/ARB & All & $66.7(100)$ & $59.3(89)$ & $77.3(116)$ & +10.6 \\
\hline Elective & & 77.1 (54) & $65.7(46)$ & $8 \mathrm{I} .4(57)$ & +4.3 \\
\hline Emergent & & $57.5(46)$ & $53.8(43)$ & 73.8 (59) & +16.3 \\
\hline Statin & All & $71.3(107)$ & $82(123)$ & $85.3(128)$ & +14 \\
\hline Elective & & $8 \mathrm{I} .4(57)$ & $85.7(60)$ & $87.1(61)$ & +5.7 \\
\hline Emergent & & $62.5(50)$ & $78.8(63)$ & $83.8(67)$ & +21.3 \\
\hline All three & All & $40.7(6 \mathrm{I})$ & 44.7 (67) & $60.7(91)$ & +20 \\
\hline Elective & & $52.9(37)$ & $50(35)$ & $64.3(45)$ & +11.4 \\
\hline Emergent & & $30(24)$ & $40(32)$ & $57.5(46)$ & +27.5 \\
\hline
\end{tabular}

Notes: aClinically acceptable reasons as determined by expert clinician panel; bomparing patients on class of medication prior to admission vs patient on class of medication at discharge, or not on medication with reason.

Abbreviations: ACEI, angiotensin converting enzyme; ARB, angiotensin receptor blockers; vs, versus. 
Table 3 Patients with clinically acceptable reasons for nonprescription of risk factor modification treatment

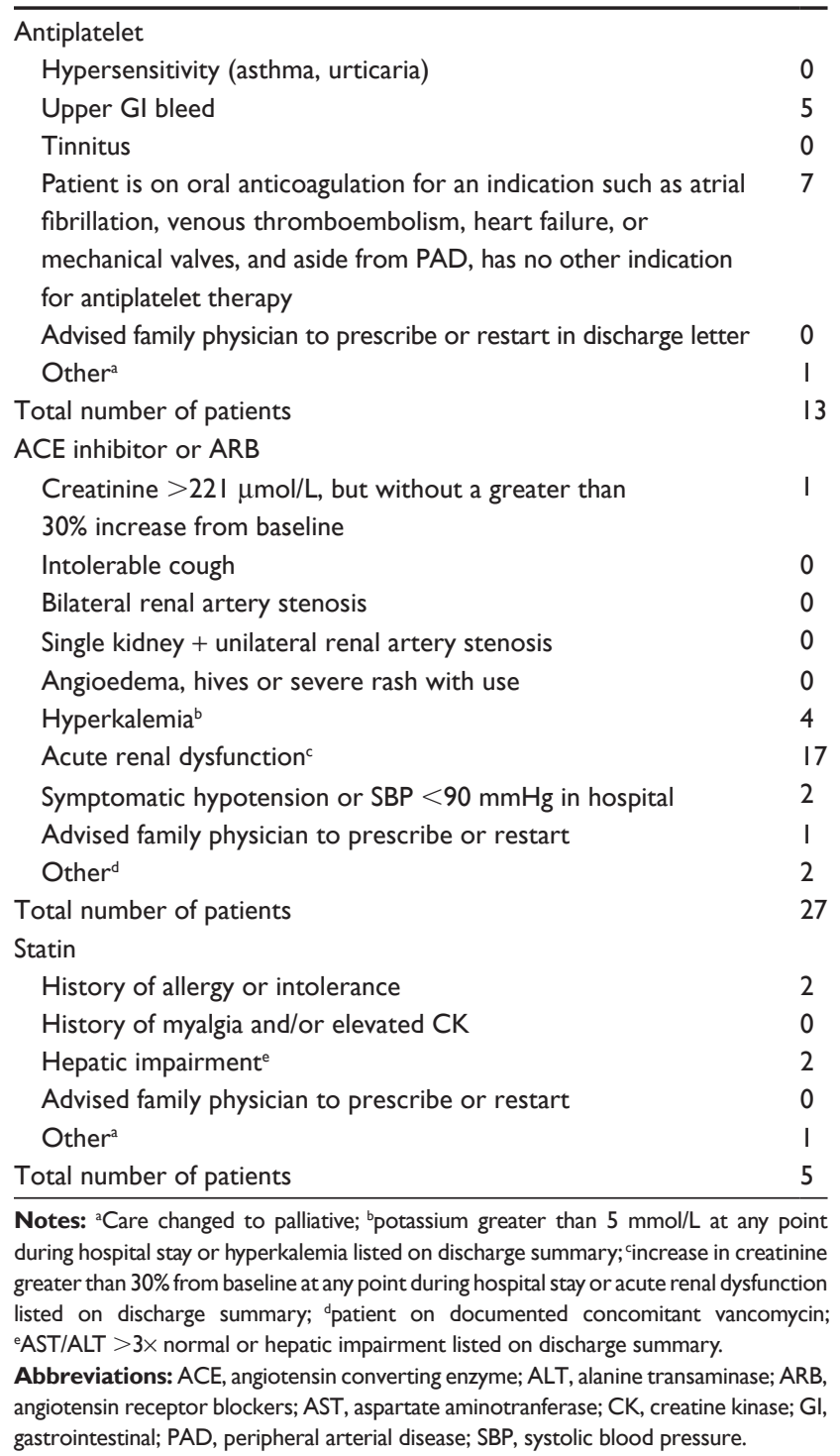

based pharmacologic therapies compared to preadmission; however, there is still room for improvement. A high proportion of our patients with PAD were discharged on an antiplatelet agent and statin, yet ACE inhibitors and ARBs appear to be underprescribed.

Platelets have a key role in the pathogenesis of atherosclerosis; antiplatelet drugs are recommended in the management of patients with PAD due to their efficacy in ischemic heart disease and cerebrovascular disease. ${ }^{5}$ However, in our population, only $64 \%$ of patients admitted to the vascular surgery service were on an antiplatelet prior to admission. This low rate is not likely due to patients holding aspirin preoperatively for elective procedures, as this is not standard practice at our institution. On discharge, $82.7 \%$ of patients left hospital on an antiplatelet. This number increases to $91.3 \%$ when we also consider patients who had a clinically acceptable reason for not being on antiplatelet therapy (Table 3 ). Given the retrospective nature of this study, we were unable to determine with confidence if the patients with a history of GI bleed would be suitable for current antiplatelet therapy. As such, history of GI bleed was considered to be an acceptable reason for antiplatelet omission for the purposes of this study. The observed overall improvement in care is substantial; however, almost $10 \%$ of patients were discharged without antiplatelet therapy and no clear reason for the omission.

In 2003, a subgroup analysis of patients with PAD was published from the Heart Outcomes Prevention Evaluation (HOPE) trial. ${ }^{14}$ Ramipril $10 \mathrm{mg}$ per day was shown to reduce the risk of a cardiovascular event by $25 \%$ in patients with symptomatic PAD. The HOPE trial also implied that ACE inhibitors are likely to benefit PAD patients with borderline or normal blood pressure. ${ }^{5}$ Historically, and in spite of the

Table 4 Prescribed risk factor modification therapy, stratified by gender

\begin{tabular}{|c|c|c|c|c|}
\hline Patient gender & $\begin{array}{l}\text { Patient on class of } \\
\text { medication prior } \\
\text { to admission } \%(\mathrm{n})\end{array}$ & $\begin{array}{l}\text { Patient on class } \\
\text { of medication at } \\
\text { discharge } \%(n)\end{array}$ & $\begin{array}{l}\text { Patient on class of medication } \\
\text { at discharge, OR not on } \\
\text { medication with reason }{ }^{\mathrm{a}} \%(\mathrm{n})\end{array}$ & $\begin{array}{l}\text { Percent } \\
\text { improved }^{\text {b }}\end{array}$ \\
\hline \multicolumn{5}{|l|}{ Antiplatelet } \\
\hline Female $(n=50)$ & $64(32)$ & $80(40)$ & $94(47)$ & +30 \\
\hline Male $(n=100)$ & $59(59)$ & $84(84)$ & $90(90)$ & +31 \\
\hline \multicolumn{5}{|l|}{ ACE inhibitor/ARB } \\
\hline Female & $74(37)$ & $58(29)$ & $78(39)$ & +4 \\
\hline Male & $63(63)$ & $60(60)$ & 77 (77) & +14 \\
\hline \multicolumn{5}{|l|}{ Statin } \\
\hline Female & $66(33)$ & $72(36)$ & $76(38)$ & +10 \\
\hline Male & $74(74)$ & $87(87)$ & $90(90)$ & +16 \\
\hline \multicolumn{5}{|l|}{ All three } \\
\hline Female & $42(21)$ & $40(20)$ & $56(28)$ & +14 \\
\hline Male & $40(40)$ & $47(47)$ & $63(63)$ & +23 \\
\hline
\end{tabular}

Notes: a Clinically acceptable reasons as determined by expert clinician panel; bomparing patients on class of medication prior to admission vs at discharge or not on medication with reason.

Abbreviations: ACE, angiotensin converting enzyme; ARB, angiotensin receptor blockers; vs, versus. 
existing evidence, ACE inhibitors and ARBs have been the most challenging class of medications to prescribe for patients with PAD. It is unknown if this is due to the greater number of contraindications in this medically complex population, or because clinicians have concerns regarding ongoing monitoring of renal function and blood pressure. In our population, $66.7 \%$ of patients were admitted to hospital on an ACE inhibitor or ARB, which is a higher percentage than patients admitted on an antiplatelet agent. On discharge, $59.3 \%$ of patients were discharged on either an ACE inhibitor or ARB. When including patients who were considered ineligible for ACE inhibitor or ARB therapy, the percentage of patients on appropriate therapy increases to $77.3 \%$. In other words, 27 patients out of the entire population (18\%) had a clinically acceptable reason for not being prescribed an ACE inhibitor or ARB at discharge. The most common reason was acute renal failure while in hospital, and we hypothesize this is why actual prescription rates decreased after admission. This highlights that this population is at considerable risk of acute renal failure, and consequently adherence to guidelinerecommended therapy with ACE inhibitors or ARBs is challenging. It also highlights that the goal of achieving $100 \%$ compliance with the guideline recommendation for the use of these agents may not be practical. For the purpose of this study, if an episode of acute renal failure occurred at any point during admission, this was considered sufficient reason to withhold ACE inhibitor therapy for the duration of the admission. Many admissions are too short to provide enough time for adequate renal recovery. Certainly this reason is no longer valid once stable renal function has been established. It would be of interest to follow these patients as outpatients to assess if and when an ACE inhibitor is initiated. This is an area for future research.

There is strong evidence to support the lowering of serum cholesterol with the use of statins as a primary therapy to reduce the likelihood of vascular events. Pleotropic effects of statins include anti-inflammatory properties, improved endothelial dependent vasodilation of peripheral arteries, and angiogenesis promotion. ${ }^{5}$ On admission, more patients were on a statin $(71.3 \%)$ than an antiplatelet agent or ACE inhibitor/ARB. At discharge, $82 \%$ of patients were on a statin.

We found that $7.7 \%$ of patients screened were excluded due to lack of electronic documentation (no BPMH, no discharge summary, or no discharge medication list). This is likely due to a number of factors: short weekend admissions may not be seen by a pharmacist and thus may not have a BPMH; very short admissions (less than 24 hours) do not always result in the generation of an electronic discharge summary; and also, occasional user error may result in documents not being saved in the computer system. These are inherent limitations related to retrospective chart reviews.

As seen in Table 1, many PAD patients have multiple comorbidities, and this may contribute to the difficulties in prescribing risk-modifying medications. This also implies, however, that these patients stand to benefit most significantly from implementing such therapies. A high proportion of patients seen by the service had documented coronary artery disease $(56.7 \%)$. The prevalence of diabetes was $40.7 \%$. This number is in line with other studies both in Ireland and in Quebec which document a prevalence of diabetes in the PAD population between $20 \%-30 \% .{ }^{8,9}$ Chronic kidney disease was also common (22\%), which likely contributes to the difficulties in prescribing ACE inhibitors and ARBs.

In comparison with emergent admissions, a higher proportion of elective patients were on risk-modifying therapies. This was anticipated as patients admitted for elective surgery would have had prior contact with healthcare providers and therefore opportunity for interventions. These patients would likely have been assessed for PAD by their family doctor and seen in the preadmission clinic prior to admission. At the time of admission, $77.1 \%$ of elective patients were on an antiplatelet, $77.1 \%$ were on an ACE inhibitor or ARB, and $81.4 \%$ were on a statin. These rates were lower in patients admitted emergently, possibly because emergent patients may be presenting with their first diagnosis of PAD. It is encouraging that patients admitted emergently had a substantial improvement in therapy at the time of discharge. For example, in emergent patients, there was a $38.8 \%$ increase in antiplatelet therapy use, $16.3 \%$ increase in ACE inhibitor or $\mathrm{ARB}$ use, and a $21.3 \%$ increase in statin use when patients who were not on therapy for an acceptable reason were included in the patient count.

Secondary endpoints of the study included describing the common reasons for not prescribing risk factor modification therapy. In addition to the list of clinically acceptable reasons developed by our experts, there were only two additional documented reasons identified through the process of chart review - patients whose care changed from active to palliative and ACE inhibitor/ARB held due to concerns of renal impairment while on concomitant vancomycin therapy. We were able to determine an acceptable reason for not being prescribed an antiplatelet in 13 patients, an ACE inhibitor or ARB in 27 patients, and a statin in five patients. It is clear that clinicians underprescribe these classes of medications 
for patients with PAD, even in patients who do not have a specific contraindication.

The influence of gender on prescription rates was also analyzed. There were twice as many men as women in our study population. Prescription rates of antiplatelets and ACE inhibitors/ARBs at discharge were similar for both genders. However, there were differences in rates of prescription of statins. Only $72 \%$ of women were discharged on a statin, compared to $87 \%$ of men. When including patients not prescribed a statin for a clinically acceptable reason, the difference between men and women $(90 \%$ for men, $76 \%$ for women) persisted. This is interesting when compared to the results from Pâquet et al where treatment rates with statins for men with PAD discharged from a tertiary teaching hospital were higher than women from the same population (49.1\% vs $46.6 \%$, respectively). ${ }^{8}$ More research is needed to understand these differences in prescribing patterns.

Because this was a retrospective chart review, the authors relied on the discharge summary medication list as the sole source of information regarding discharge medications. This was a good reference to use, as discharge medication reconciliation is achieved for over $80 \%$ of patients on this unit; however, there was no way to verify patient compliance with this list of medications after discharge.

This study is subject to the limitations inherent to retrospective chart reviews. Patients who may have otherwise been eligible may not have met inclusion criteria simply due to lack of documentation. This study took place during a specific 6-month timeframe in 2010 and may or may not be generalizable to other periods in time. The acceptable reasons for not prescribing a medication at discharge were gathered from the chart. However, information regarding reasons for nonprescribing prior to admission were not available, so it was not possible to collect this information. The list of clinically acceptable reasons was developed specifically for this study and had not been previously validated. Given that there were only two additional acceptable reasons discovered during chart review, the list can be considered comprehensive. PAD guidelines also recommend other management strategies including hypertension, diabetes, and smoking cessation therapy. This study was not designed to address these components of PAD management. The sample size is not large enough to allow for tests of statistical significance. However, in terms of clinical significance, there was a clear increase in the numbers of patients receiving secondary prevention therapies. In addition, the sample size of 150 is a large and informative number which allows for a descriptive quality of care assessment that is clinically relevant and is based on the needs and resources of the institution.

Although it is clear that admission to the vascular surgery service increased the number of patients on appropriate therapy, $39.3 \%$ of patients were, in fact, discharged from hospital without all three therapies prescribed. This suggests there are barriers to providing optimal care. While this study did not specifically investigate these barriers, the authors speculate the following reasons: some admissions were short, with limited opportunity for initiating therapy; some admissions occurred over the weekend, when staff is limited and less likely to be performing a full medication review; and there may be some discrepancy among physicians regarding who is responsible for initiating such therapies. Some may think this is the responsibility of the primary care physician, not the surgical team. Additionally, this population is very complex. It may be difficult to assess the appropriateness of certain medications in a population with multiple comorbidities, and perhaps more prescriber education is required. Future research should focus on determining which barriers exist and developing methods and tools for overcoming these barriers.

Other areas for research include reviewing the study population in 12 to 24 months to gain an understanding of long-term adherence rates months after in-patient admission. This study also highlights that areas of improvement for the vascular surgery service exist. Opportunities for intervention within the program need to be identified, but also at other points of contact with health care providers in the community prior to presenting to a tertiary care center.

\section{Conclusion}

In-patient admission to our vascular surgery service correlates with clinically important improvement in pharmacological risk factor management. This improvement occurs most dramatically in the emergent population, and is gender neutral. Nevertheless, some patients are still not receiving evidence-based treatment at discharge. This warrants consideration of further strategies to improve PAD guideline adherence, in both the community and the vascular surgery service.

\section{Disclosure}

The authors declare no conflicts of interest in this work.

\section{References}

1. Lovell M, Harris K, Forbes T, et al; Peripheral Arterial Disease Coalition. Peripheral arterial disease: lack of awareness in Canada. Can J Cardiol. 2009;25(1):39-45. 
2. Weitz JI, Byrne J, Clagett GP, et al. Diagnosis and treatment of chronic arterial insufficiency of the lower extremities: a critical review. Circulation. 1996;94(11):3026-3049.

3. Hirsch AT, Haskal ZJ, Hertzer NR, et al. ACC/AHA 2005 guidelines for the management of patients with peripheral arterial disease (lower extremity, renal, mesenteric, and abdominal aortic): a collaborative report from the American Association for Vascular Surgery/Society for Vascular Surgery, Society for Cardiovascular Angiography and Interventions, Society for Vascular Medicine and Biology, Society of Interventional Radiology, and the ACC/AHA Task Force on Practice Guidelines (Writing Committee to Develop Guidelines for the Management of Patients With Peripheral Arterial Disease). J Am Coll Cardiol. 2006;47(6):e1-e192.

4. Rooke TW, Hirsch AT, Misra S, et al; Society for Cardiovascular Angiography and Interventions; Society of Interventional Radiology; Society for Vascular Medicine; Society for Vascular Surgery. 2011 ACCF/AHA Focused Update of the Guideline for the Management of Patients With Peripheral Artery Disease (updating the 2005 guideline): a report of the American College of Cardiology Foundation/American Heart Association Task Force on Practice Guidelines. J Am Coll Cardiol. 2011;58(19):2020-2045.

5. Abramson BL, Huckell V, Anand S et al. Canadian Cardiovascular Society Consensus Conference: peripheral arterial disease - executive summary. Can J Cardiol. 2005;21(12):997-1006.

6. Bell AD, Roussin A, Cartier R, et al; Canadian Cardiovascular Society. The use of antiplatelet therapy in the outpatient setting: Canadian Cardiovascular Society guidelines. Can J Cardiol. 2011;27 Suppl A: S1-S59.
7. Pande RL, Perlstein TS, Beckman JA, Creager MA. Secondary prevention and mortality in peripheral artery disease: National Health and Nutrition Examination Study, 1999 to 2004. Circulation. 2011;124(1):17-23.

8. Pâquet M, Pilon D, Tétrault JP, Carrier N. Protective vascular treatment of patients with peripheral arterial disease: guideline adherence according to year, age and gender. Can J Public Health. 2010;101(1): 96-100.

9. Sunderland M, De Jong M, Bates D. Vascular protection in patients with diabetes admitted for vascular surgery in a canadian tertiary care hospital: pilot study. Can J Hosp Pharm. 2013;66(4):227-232.

10. Coveney AP, O'Brien GC, Fulton GJ. ACE up the sleeve - are vascular patients medically optimized? Vasc Health Risk Manag. 2011;7: $15-21$.

11. University Health Network Corporate Intranet. Program Groupings. Peter Munk Cardiac Centre: Vascular Surgery [webpage on the Internet]. Available at: https://intranet.remote.uhn.ca/cvpn/IpAnkAjrG_1etKhCof6x18jmOkD/departments/programs/heart/vascular_surgery.asp. Accessed January 19, 2014.

12. CritiCall Ontario [webpage on the Internet]. Available at http://criticall. org. Accessed June 16, 2012.

13. The ONTARGET Investigators; Yusuf S, Teo KK, et al. Telmisartan, ramipril or both in patients at high risk for vascular events. $N$ Engl J Med. 2008;358(15):1547-1559.

14. Mehler PS, Coll JR, Estacio R, Esler A, Schrier RW, Hiatt WR. Intensive blood pressure control reduces the risk of cardiovascular events in patients with peripheral arterial disease and type 2 diabetes. Circulation. 2003;107(5):753-756.
Vascular Health and Risk Management

\section{Publish your work in this journal}

Vascular Health and Risk Management is an international, peerreviewed journal of therapeutics and risk management, focusing on concise rapid reporting of clinical studies on the processes involved in the maintenance of vascular health; the monitoring, prevention and treatment of vascular disease and its sequelae; and the involvement of

\section{Dovepress}

metabolic disorders, particularly diabetes. This journal is indexed on PubMed Central and MedLine. The manuscript management system is completely online and includes a very quick and fair peer-review system, which is all easy to use. Visit http://www.dovepress.com/ testimonials.php to read real quotes from published authors. 